\title{
Sequential multiplex PCR for identifying pneumococcal capsular serotypes from south- Saharan African clinical isolates
}

\begin{abstract}
Correspondence
Bernard Beall

BBeall@cdc.gov
\end{abstract}

Received 16 April 2007

Accepted 24 May 2007

\author{
Luis Morais, ${ }^{1}$ Maria da Glória Carvalho, ${ }^{2}$ Anna Roca, ${ }^{1,3}$ Brendan Flannery, ${ }^{2}$ \\ Inacio Mandomando, ${ }^{1,4}$ Montserrat Soriano-Gabarró, ${ }^{2}$ Betuel Sigauque, ${ }^{1,4}$ \\ Pedro Alonso ${ }^{1,3}$ and Bernard Beall ${ }^{2}$ \\ ${ }^{1}$ Centro de Investigação em Saúde da Manhiça (CISM), Ministerio de Saúde, CP1929 Maputo, \\ Mozambique \\ ${ }^{2}$ Respiratory Diseases Branch, Division of Bacterial Diseases, Centers for Disease Control and \\ Prevention, Atlanta, GA 30333, USA \\ ${ }^{3}$ Centre de Salut Internacional (CSI), Hospital Clinic/IDIBAPS, Universitat de Barcelona, \\ Barcelona, Spain \\ ${ }^{4}$ Instituto Nacional de Saúde Ministério de Saúde, Maputo, Mozambique
}

A serial multiplex PCR approach was reformulated for pneumococcal serotyping to test 153 clinical isolates from children in Mozambique. This approach identified serotypes in 139 (90.8\%) of 153 isolates; 126 (82.4\%) were identified within two reactions. This approach in developing countries would require minimal training and could provide useful serotype information without requiring transport of specimens.

\section{INTRODUCTION}

Streptococcus pneumoniae is among the leading causes of childhood death in Africa (Berkley et al., 2005). More data are needed on pneumococcal serotype distribution in Africa to advocate the introduction of appropriately formulated pneumococcal conjugate vaccines and to conduct surveillance for replacement disease after vaccine introduction. Few reference laboratories in Africa perform pneumococcal serotyping using the Quellung reaction, the gold standard for serotyping. Research laboratories send pneumococcal isolates to international reference laboratories for serotyping at considerable expense. Here, we evaluated a serial multiplex PCR approach (Pai et al., 2006) for identification of serotypes or serogroups of pneumococcal isolates from hospitalized children in Mozambique. We evaluated the flexibility of this method by altering combinations of serotype specificities and determined that our primer pairs were specific for corresponding serotypes from isolates recovered in this region.

\section{METHODS}

Pneumococci ( $n=163)$ were isolated from blood, cerebrospinal fluid or middle ear exudate from children under 15 years of age admitted to Manhiça district hospital between June 2003 and May 2006; $66 \%$ were from children less than 2 years old. Automated blood cultures

Abbreviation: CDC, Centers for Disease Control and Prevention. were performed following hospital admission for all children less than 2 years of age and for children aged 2-14 years with axillary temperatures $>39{ }^{\circ} \mathrm{C}$ as described previously (Roca et al., 2006; Valles et al., 2006). $\alpha$-Haemolytic colonies were tested for optochin susceptibility in the microbiology laboratory of the Centro de Investigaçao em Saúde da Manhiça (CISM-Manhiça Health Research Center) to differentiate $S$. pneumoniae from other $\alpha$-haemolytic streptococci, and pneumococcal isolates were stored at $-70{ }^{\circ} \mathrm{C}$ in $10 \%$ skimmed milk. Isolates were recovered prior to transportation and shipped in STGG medium at $4{ }^{\circ} \mathrm{C}$ as described previously (Valles et al., 2006).

Pneumococcal isolates were conventionally serotyped using the Centers for Disease Control and Prevention (CDC) procedure and multiplex PCR was performed following a published protocol (Pai et al., 2006). Combinations of primer sets in the first two reactions were designed to identify $80 \%$ of invasive pneumococci based on African serotype surveillance data (Hausdorff et al., 2000; Valles et al., 2006). Each of the seven reactions included four primer pairs targeting different serotype-specific sequences (with an additional primer pair in reaction 6; see Table 1), plus the internal positive control for a $160 \mathrm{bp}$ conserved region in the pneumococcal cps operon. We modified the order of primers proposed previously for African isolates (Pai et al., 2006) in order to improve visualization of results: primers for serotype 5 were included in PCR 1 and primers for serotype 1 were included in PCR 2 (Fig. 1). DNA extraction, PCRs and electrophoresis were performed as described previously (Pai et al., 2006), except that serotype combinations were formulated to avoid PCR products with differences in sizes of $<50 \mathrm{bp}$ to allow better resolution in $2 \%$ agarose gels (see Fig. 1). Reactions in which the positive control target was not amplified were repeated. Serotypes were retested by the Quellung reaction when a discordant serotype was identified by multiplex PCR. 
Table 1. Serotyping results from sequential multiplex PCRs for the 153 pneumococcal isolates from Mozambique

\begin{tabular}{|c|c|c|c|c|}
\hline $\begin{array}{l}\text { Serotypes included in } \\
\text { multiplex PCR, by } \\
\text { reaction }^{\star}\end{array}$ & $\begin{array}{l}\text { Expected PCR } \\
\text { product (bp) }\end{array}$ & $\begin{array}{l}\text { No. of targets } \\
\text { amplified }\end{array}$ & $\begin{array}{l}\text { Serotype by Quellung } \\
\text { reaction (no. of positive } \\
\text { isolates) }\end{array}$ & $\begin{array}{l}\text { Isolates (\%) } \\
\quad(n=153)\end{array}$ \\
\hline PCR 1 & & 68 & & 45 \\
\hline 5 & 362 & 16 & $5(16)$ & \\
\hline $6 \mathrm{~A} / 6 \mathrm{~B}$ & 250 & 38 & $6 \mathrm{~A}(22), 6 \mathrm{~B}(15), 24 \mathrm{~F} \dagger(1)$ & \\
\hline $19 \mathrm{~A}$ & 478 & 7 & $19 \mathrm{~A}(7)$ & \\
\hline $19 \mathrm{~F}$ & 304 & 7 & 19F (6), 9L $\ddagger(1)$ & \\
\hline PCR 2 & & 57 & & 37 \\
\hline 1 & 280 & 32 & $1(32)$ & \\
\hline $7 \mathrm{~F} /(7 \mathrm{~A})$ & 826 & 1 & $7 \mathrm{~F}(1)$ & \\
\hline 14 & 208 & 12 & $14(12)$ & \\
\hline $23 \mathrm{~F}$ & 384 & 12 & $23 \mathrm{~F}(12)$ & \\
\hline PCR 3 & & 11 & & 7 \\
\hline 4 & 430 & 4 & $4(4)$ & \\
\hline $9 \mathrm{~V} /(9 \mathrm{~A})$ & 753 & 4 & $9 \mathrm{~V}(4)$ & \\
\hline $12 \mathrm{~F} /(12 \mathrm{~A})$ & 376 & 1 & $12 \mathrm{~F}(1)$ & \\
\hline $22 \mathrm{~F} /(22 \mathrm{~A})$ & 643 & 2 & $22 \mathrm{~A}(1), 22 \mathrm{~F}(1)$ & \\
\hline PCR 4 & & 2 & & 1 \\
\hline 3 & 371 & 0 & - & \\
\hline $16 \mathrm{~F}$ & 988 & 0 & - & \\
\hline $18 \mathrm{C} /(18 \mathrm{~A}, 18 \mathrm{~B}, 18 \mathrm{~F})$ & 573 & 1 & $18 \mathrm{C}(1)$ & \\
\hline $35 \mathrm{~B}$ & 677 & 1 & 35B (1) & \\
\hline PCR 5 & & 0 & & 0 \\
\hline 8 & 294 & 0 & - & \\
\hline $15 B / 15 C$ & 496 & 0 & - & \\
\hline 31 & 701 & 0 & - & \\
\hline $38 /(25 \mathrm{~F})$ & 574 & 0 & - & \\
\hline PCR 6 & & 1 & & 1 \\
\hline $10 \mathrm{~A}$ & 628 & 1 & $10 \mathrm{~A}(1)$ & \\
\hline $11 \mathrm{~A} /(11 \mathrm{D})$ & 463 & 0 & - & \\
\hline $33 \mathrm{~F} /(33 \mathrm{~A}, 37)$ & 338 & 0 & - & \\
\hline 34 & 408 & 0 & - & \\
\hline $35 \mathrm{~F} /(47 \mathrm{~F})$ & 517 & 0 & - & \\
\hline PCR 7 & & 1 & & 1 \\
\hline $7 \mathrm{C} /(7 \mathrm{~B}, 40)$ & 260 & 1 & 7C (1) & \\
\hline $15 \mathrm{~A}$ & 436 & 0 & - & \\
\hline $17 \mathrm{~F}$ & 693 & 0 & - & \\
\hline 20 & 514 & 0 & - & \\
\hline $\begin{array}{l}\text { Not typed }-c p s A- \\
\text { positive only }\end{array}$ & 160 & 13 & $\begin{array}{l}\text { 9L (2), } 13(1), 23 \mathrm{~A}(2) \\
24 \mathrm{~F}(4), 29(2), 33 \mathrm{D}(1) \\
33 \mathrm{~B}(1)\end{array}$ & 8 \\
\hline
\end{tabular}

${ }^{\star}$ Parentheses indicate rarely occurring serotypes that are not resolved by this method.

$\dagger$ Repeat of Quellung reaction identified the serotype as 6B.

$\ddagger$ Repeat of Quellung reaction identified the serotype as 19F.

\section{RESULTS AND DISCUSSION}

Of the 163 isolates stored in the CISM laboratory, five failed to grow. Of the 158 isolates transported to the Streptococcus Laboratory at the US CDC, two isolates failed to grow and three isolates were not $S$. pneumoniae. Among the 153 pneumococcal isolates recovered for serotyping, 143 were from blood, eight from cerebrospinal fluid and two from purulent ear fluid. PCR 1 identified 68 (44.4\%) of the 153 isolates. Sixty-six (97.1\%) of the 68 PCR 1 results were consistent with the Quellung reaction results. For the two discrepant results, the PCR 1 results were found to be correct after retyping by Quellung (Table 1). PCR 2 was performed for the remaining 85 isolates that had amplified only the positive control cpsA locus in PCR 1. PCR 2 identified serotypes for $57(67.1 \%)$ of the 85 

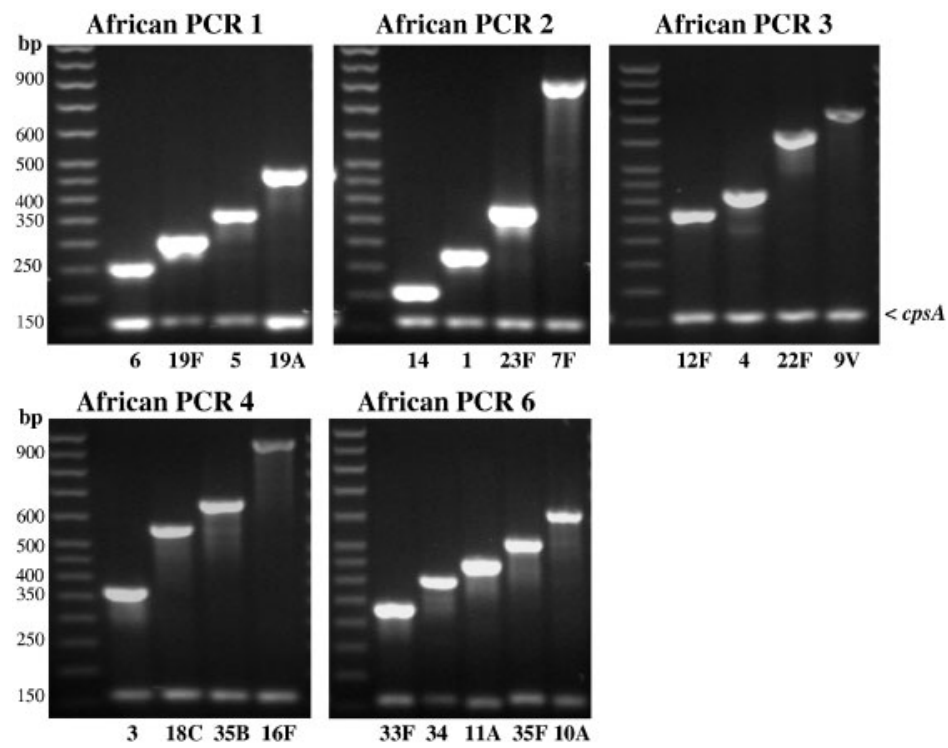

Fig. 1. Multiplex PCRs 1, 2, 3, 4 and 6 for $S$. pneumoniae serotyping in Africa. PCRs 5 and 7 were as described in Pai et al. (2006). The position of the cpsA fragment is indicated by an arrowhead.

isolates and all were concordant with Quellung reaction results. Overall, the first two PCRs identified 125 (81.7\%) of 153 isolates from hospitalized children in Mozambique, whilst PCRs 3-7 identified serotypes for 15 additional isolates $(9.8 \%)$. After seven sequential PCRs, only 13 isolates $(8.5 \%)$ were not assigned serotypes. Only one reaction failed to amplify the positive control and therefore needed to be repeated.

Classical serotyping at the CDC required 360 screening reactions with pools of antisera, 437 latex agglutination reactions and 305 Quellung reactions to serotype the 153 pneumococcal isolates. In contrast, only 331 PCRs were required to type $140(91.5 \%)$ of the 153 isolates. In summary, serial multiplex PCR proved useful for identification of the principal serotypes causing pneumococcal disease among children in Mozambique. PCR correctly identified all serotype 1 and 5 isolates, which have been found previously in our hospital to be responsible for $>50 \%$ of invasive pneumococcal disease during a 12month period (Valles et al., 2006). Combinations of primers formulated to identify pneumococcal serotypes more common in developing countries than in the USA (Pai et al., 2006) identified $82 \%$ of the isolates in this sample in the first two multiplex reactions. Even for locations where a lower percentage of pneumococcal isolates are serotypes 1 and 5, primers included in all seven reactions used for this study are likely to identify $\sim 90 \%$ of the serotypes causing disease in children. The serial PCR procedure required minimal training and no prior experience with PCR for efficient and accurate serotype surveillance, and was far less time-consuming than classical Quellung reaction-based serotyping. The major disadvantage of the current PCR approach was the inability to determine all serotypes (see 'Not typed - cpsApositive only', in Table 1). Other disadvantages included the inability to resolve the commonly occurring serotypes
$6 \mathrm{~A}$ and $6 \mathrm{~B}$, and the inability to resolve certain serotypes from rarely occurring serotypes within the same serogroup (22F/22A; Table 1).

The inclusion of the positive-control primers in each reaction reliably identified sample preparation problems so that reactions that failed to amplify a product could be repeated. With the widespread availability of conventional PCR machines, this approach can be used to type pneumococcal isolates at microbiology laboratories that lack type-specific pneumococcal antisera. The work presented here, and in the accompanying report dealing with Brazilian isolates (Dias et al., 2007), is consistent with the notion that the included primer sets are specific for the corresponding serotypes from diverse collections of isolates collected in developing or newly industrialized countries. Major advantages of this approach include the potential to type small numbers of isolates at moderate expense, prompt identification of predominant serotypes for case investigations, and a reduction in the number of isolates requiring transport to reference laboratories for serotyping. Further modifications of the method will be described at the CDC Streptococcus website (www.cdc.gov/ncidod/biotech/strep/PRC.htm).

\section{ACKNOWLEDGEMENTS}

The Manhiça Health Research Center (CISM) receives core funding from the Spanish Agency for International Cooperation (Ministry of Foreign Affairs, Spain). Collection of pneumococcal isolates was funded by grants from WHO (TSA 18-181-1200-HQ/02/186921) and the Children's Vaccine Program at the Program for Appropriate Technology in Health, Seattle, WA, USA (GAT.770-790-01350-LPS). The Hospital Clinic receives support from the RECESP-C03/04 (Red Centros de Investigación Cooperativa en Epidemiología y Salud Pública - Ministry of Health, Spain). The authors are grateful to Mariano Sitaúbe of the bacteriology laboratory, CISM, for culturing and identifying bacteria, Delois Jackson and Terry Thompson, for classical serotyping, and Robert Gertz, for assistance with PCR serotyping. The 
authors thank colleagues at Manhiça District Hospital and CISM for collecting specimens and completing questionnaires.

\section{REFERENCES}

Berkley, J. A., Lowe, I., Mwangi, B. S., Williams, T., Bauni, E., Mwarumba, S., Ngetsa, C., Slack, M. P., Njenga, S. \& other authors (2005). Bacteremia among children admitted to a rural hospital in Kenya. N Engl J Med 352, 39-47.

Dias, C. A., Teixeira, L. M., Carvalho, Mda. G. \& Beall, B. (2007). Sequential multiplex PCR for determining capsular serotypes of pneumococci recovered from Brazilian children. J Med Microbiol 56, 1185-1188.

Hausdorff, W. P., Bryant, J., Paradiso, P. R. \& Siber, G. R. (2000). Which pneumococcal serogroups cause the most invasive disease: implications for conjugate vaccine formulation and use, part I. Clin Infect Dis 30, 100-121.

Pai, R., Gertz, R. E. \& Beall, B. (2006). Sequential multiplex PCR approach for determining capsular serotypes of Streptococcus pneumoniae isolates. J Clin Microbiol 44, 124-131.

Roca, A., Sigauque, B., Quinto, L., Mandomando, I., Valles, X., Espasa, M., Abacassamo, F., Sacarlal, J., Macete, E. \& other authors (2006). Invasive pneumococcal disease in children $<5$ years of age in rural Mozambique. Trop Med Int Health 11, 1422-1431.

Valles, X., Flannery, B., Roca, A., Mandomando, I., Sigauque, B., Sanz, S., Schuchat, A., Levine, M., Soriano-Gabarro, M. \& Alonso, P. (2006). Serotype distribution and antibiotic susceptibility of invasive and nasopharyngeal isolates of Streptococcus pneumoniae among children in rural Mozambique. Trop Med Int Health 11, 358-366. 